\title{
Average Per-user Rate for MIMO Systems with SDM-FDPS
}

\author{
Youjia Chen, Zihuai Lin \\ School of Physics and OptoElectronics Technology \\ Fujian Normal University \\ Fuzhou, Fujian, China \\ chenyoujia@fjnu.edu.cn, zihuai@ee.usyd.edu.au
}

\author{
Pei Xiao, Merhdad Dianati \\ Centre for Communication Systems Research \\ University of Surrey \\ Surrey, United Kingdom \\ \{p.xiao, m.dianati\}@surrey.ac.uk
}

\begin{abstract}
In this paper, we introduce the concept of average per-user rate to the multiuser Multiple-Input, Multiple-Output (MIMO) system with the frequency domain packet scheduler (FDPS) at base stations, which provides an estimate of the rate that the system could provide for each admitted user. The proposed admission control is designed by comparing the user's quality of service $(\mathrm{QoS})$ requirements with the transmission rate that the system can offer. The analytical model is based on the generalized 3GPP LTE downlink transmission for which two Spatial Division Multiplexing (SDM) multiuser MIMO schemes are investigated, namely, Single User (SU) and Multi-user (MU) MIMO schemes. The main contribution of this paper is the derivation of the achievable rate for each user in the SDM MIMO systems based on a mathematical model of the Signal to Interference plus Noise Ratio (SINR) distribution with the frequency domain packet scheduler. The achievable rate provides insights into the system's performance from a different perspective.
\end{abstract}

\section{INTRODUCTION}

In 3GPP Long Term Evolution (LTE) (also known as Evolved-UMTS Terrestrial Radio Access (E-UTRA)), Multiple-Input Multiple-Output (MIMO) and Orthogonal Frequency Division Multiple Access (OFDMA) have been selected for downlink transmission [1]. Both Spatial Division Multiplexing (SDM) and Frequency Domain Packet Scheduling (FDPS) have been proposed. In [2], it is shown that the MIMO schemes with combined SDM and FDPS can further enhance the system performance.

Both open loop and closed loop $\mathrm{MIMO}^{1}$ are considered as possible solutions in 3GPP LTE. However, the closed loop solution provides both diversity and array gains, and hence a superior performance [3]. And the use of linear precoding has been widely studied as a closed loop scheme, due to its simplicity and robust performance [4]. In [5], Dianati et al. proposed an analytical model for the average per-user rate to estimate the resource that the system could provide for each user in this system. Meanwhile, an admission control policy is proposed based on this rate: if the required resources could be satisfied and does not influence the existing users' requirements, the new user will be admitted into the system. Unlike the traditional performance metrics, such as total

\footnotetext{
${ }^{1}$ Open loop and closed loop MIMO correspond to the MIMO systems without and with channel state information at the transmitter, respectively [1].
}

system throughput, the use of average per-user rate presents a novel approach to assessing the system performance from the perspective of its service offered to each user.

In this paper, we derive the achievable average rate for each user in the systems under question based on the theoretical analysis of Signal to Interference plus Noise Ratio (SINR) distribution in MIMO systems with SDM-FDPS. The achievable average rate represents the long term average transmission rate the system could offer for each user. Furthermore, in order to make our schemes more practically applicable, we take the unsaturated case into consideration, where users have idle state and allow packet queues to be empty.

In the remainder of this paper, we present the multiuser SDM MIMO system model in Section II, where the FDPS algorithm is also discussed. Section III describes the achievable average per-user rate for SU-MIMO and MU-MIMO schemes respectively, based on their SINR distributions. Both open loop and closed loop MIMO schemes are considered. Furthermore, we expand this result into the unsaturated case. The analytical and numerical results are presented in Section IV. Finally, the conclusions are drawn in Section V.

\section{SYSTEM MODEL}

In this section, we describe the system model of multiuser SDM MIMO schemes for 3GPP LTE downlink transmission with packet scheduling. The basic scheduling unit in LTE is the Physical Resource Block (PRB). For the localized transmission $^{2}$ scheme, two SDM schemes are now under investigation [1], i.e., Single User (SU) MIMO and Multi-User (MU) MIMO schemes. They differ in terms of the freedom allowed to the scheduler in the spatial domain [1]. With SUMIMO scheme, only one single user can be scheduled per PRB; whereas with MU-MIMO scheme, multiple users can be scheduled per PRB, one user for each sub-stream per PRB.

The Frequency Domain (FD) scheduling algorithm considered in this work is the FD Proportional Fair (PF) [6] packet scheduling algorithm, which is being investigated under LTE. With the FD PF scheduling algorithm, the scheduler selects users at the $k$ th time slot according to $k^{*}=$

\footnotetext{
${ }^{2}$ In the localized FDMA transmission, each user's data is transmitted by consecutive subcarriers, while for the distributed FDMA transmission scheme, the user's data is transmitted by distributed subcarriers [1].
} 
$\arg \max _{k \in\{1,2, \cdots, K\}}\left\{\frac{\operatorname{SINR_{l,k}}}{\overline{S I N R_{l, k}}}\right\}$, where $S I N R_{l, k}$ is the received SINR for user $k$ at the $l$ th time slot, and $\overline{S I N R_{l, k}}$ is the average of $S I N R_{l, k}$ over a sliding window of $T_{w i n}$ time slots. In this paper, for simplicity, we only consider the case when all users in the system have equal average received SINR based on simplified assumptions similar to those made in [7]. When all users have equal average received SINR, the scheduler at the BS just selects the users with the best effective SINRs ${ }^{3}$. This assumption becomes valid when all users have roughly the same channel condition, so that the average throughput for all users are approximately the same.

The system considered here has $n_{t}$ transmit antennas at the Base Station (BS) and $n_{r}$ receive antennas for the MS in SUMIMO case, and a single receive antenna for each MS in MU-MIMO case. In the latter case, we assume $n_{r}$ MSs group together to form a virtual MIMO between BS and the group of MSs. The number of users simultaneously served on each PRB for the MU-MIMO scheme is usually limited by the number of transmitter antennas $n_{t}$. The scheduler in BS selects at most $n_{t}$ users per PRB from the $K_{T}$ active users in the cell for data transmission.

For the MU-MIMO SDM scheme with linear precoding, we use the Transmit Antenna Array (TxAA) technique [9] which is also known as the Closed Loop Transmit Diversity (CLTD) [10] in the terminology of 3GPP. In the TxAA scheme, the antenna weight vector is selected to maximize the SNR at the MS. Furthermore, we assume that the selected users can cooperate with each other and investigate the scenarios where the downlink cooperative MIMO is possible.

With a linear Minimum Mean Square Error (MMSE) receiver [11], the optimum precoding matrix under the sum power constraint can generally be expressed as $\mathbf{B}_{n}=$ $\mathbf{U}_{n} \sqrt{\boldsymbol{\Sigma}_{n}} \mathbf{V}_{n}$ [12]. Here $\mathbf{U}_{n}$ is an $n_{t} \times n_{t}$ eigenvector matrix with columns corresponding to the $n_{t}$ largest eigenvalues of the matrix $\mathbf{H}_{n} \mathbf{H}_{n}^{H}$, where $\mathbf{H}_{n}^{H}$ is the Hermitian transpose of the channel matrix $\mathbf{H}_{n}$. For Schur-Concave objective functions, $\mathbf{V}_{n} \in \mathbb{C}^{n_{t} \times n_{t}}$ is an unitary matrix, and $\boldsymbol{\Sigma}_{n}$ is a diagonal matrix with the $\eta$ th diagonal entry $\boldsymbol{\Sigma}_{n}(\eta, \eta)$ representing the power allocated to the $\eta$ th established data sub-stream, $\eta \in\left\{1,2, \cdots, n_{t}\right\}$.

\section{THE AVERAGE ACHIEVABLE PER-USER RATE}

With opportunistic scheduling, the average per-user rate for a single user in the system $\bar{R}$, which represents the long term average transmission rate of each user, can be expressed according to [5] as

$$
\bar{R}=\int_{0}^{\infty}\left[\int_{z}^{\infty} R(x) f_{X \mid Z}(x \mid Z=z) d x\right] f_{Z}(z) d z .
$$

\footnotetext{
${ }^{3}$ The unified effective SINR is defined as the equivalent single stream SINR which offers the same instantaneous (Shannon) capacity as a MIMO scheme with multiple streams [8]. Let $\gamma_{q}, q \in\{1,2, \cdots\}$, be the SINR of the $q$ th sub-stream, and $\gamma_{u}$ be the unified effective SINR, then $\log _{2}\left(1+\gamma_{u}\right)=$ $\sum_{q} \log _{2}\left(1+\gamma_{q}\right)$, so $\gamma_{u}=\prod_{q}\left(1+\gamma_{q}\right)-1$. The distribution of $\gamma_{u}$ can be derived given the distribution of $\gamma_{q}$. The purpose of introducing the unified effective SINR is to facilitate the SINR comparison between SU MIMO and MU MIMO schemes.
}

where $R(x)$ represents the achievable transmission rate as the function of the SINR value at the receiver. $X$ represents the SINR of the chosen user, $Z$ represents the maximum SINR of all other competing users who are not being served. $f_{Z}(z)$ is the Probability Density Function (PDF) of $Z$, and $f_{X \mid Z}(x \mid Z=z)$ is the conditional PDF of the selected user's SINR. Apparently, the average per-use rate $\bar{R}$ depends on the number of admitted users and the average qualities of wireless channels.

We can describe the achievable throughput as a function of SINR, e.g., $R(x)=\log _{2}(1+x)$. Based on the SINR distribution of the users and (1), the average achievable peruser rate in the LTE SDM MIMO system can be derived.

\section{A. Average Achievable Per-user Rate in SU-MIMO Scheme}

For localized downlink transmission with SU-MIMO SDM scheme [1] and FDPF algorithm under the simplifying assumptions as mentioned in Section II, in each time-slot the system will select one user whose effective SINR $\Gamma_{u}$ is the largest among all the $K_{T}$ users. In such scenario, $Z$ represents the maximum effective SINR of all the other $K_{T}-1$ competing users. Because the fading statistics for all users are independently and identically distributed. Let $F_{\Gamma_{u}}(z)$ and $f_{\Gamma_{u}}(z)$ represent the Cumulative Distribution Function (CDF) and PDF of user's effective SINR, respectively. Then, the CDF of $Z$ could be given by

$$
F_{Z}(z)=\left[F_{\Gamma_{u}}(z)\right]^{K_{T}-1} .
$$

Since $x$ is independent of $Z$, we have $f_{\Gamma_{u} \mid Z}(x \mid Z=z)=$ $f_{\Gamma_{u}}(x)$.

Using (1), the average achievable per-user rate in SU-MIMO scheme can be derived as

$$
\begin{array}{r}
\bar{R}=\int_{0}^{\infty}\left[\int_{z}^{\infty} \log _{2}(1+x) f_{\Gamma_{u}}(x) d x\right] \\
\cdot\left(K_{T}-1\right)\left[F_{\Gamma_{u}}(z)\right]^{K_{T}-2} f_{\Gamma_{u}}(z) d z .
\end{array}
$$

1) Open Loop SU-MIMO Scheme: For an uncoded MIMOOFDM system with a ZF receiver, where signals are transmitted over an uncorrelated flat Rayleigh fading channel at each subcarrier ${ }^{4}$, the SINR on the $k$ th sub-stream has a Chisquared PDF [13]. For a dual stream spatial multiplexing MIMO system with a $2 \times 2$ antenna configuration, the CDF of the unified effective SINR for the $i$ th user can be represented by $[8]$

$$
F_{\Gamma_{u}^{i}}(\gamma)=P_{r}\left(\Gamma_{u}^{i} \leq \gamma\right)=\int_{0}^{\gamma} \frac{2}{\gamma_{0}} e^{-\frac{2 x}{\gamma_{0}}}\left(1-e^{-\frac{2(\gamma-x)}{\gamma_{0}(1+x)}}\right) d x
$$

where $i \in\left\{1,2, \cdots, K_{T}\right\}, K_{T}$ is the number of active users in the cell, and $\gamma_{0}=E_{s} / N_{0}, E_{s}$ is the average transmit symbol energy per antenna and $N_{0}$ is the power spectral density of the additive white Gaussian noise.

\footnotetext{
${ }^{4}$ This is a valid assumption since the OFDM technique transforms the broadband frequency selective channel into many parallel narrow band subchannels, each of which can be treated as a flat Rayleigh fading channel.
} 
Substituting (3) and its PDF into (2), the average achievable per-user rate in the open loop SU-MIMO scheme can be derived as

$$
\begin{aligned}
\bar{R} & =\int_{0}^{\infty}\left[\int_{z}^{\infty} \log _{2}(1+x)\left(\int_{0}^{x} \frac{4}{\gamma_{0}^{2}(1+v)} e^{-\frac{2\left(x+v^{2}\right)}{\gamma_{0}(1+v)}} d v\right) d x\right] \\
& \cdot\left(K_{T}-1\right)\left[\int_{0}^{z} \frac{2}{\gamma_{0}} e^{-\frac{2 v}{\gamma_{0}}}\left(1-e^{-\frac{2(z-v)}{\gamma_{0}(1+v)}}\right) d v\right]^{K_{T}-2} \\
& \cdot\left(\int_{0}^{z} \frac{4}{\gamma_{0}^{2}(1+v)} e^{-\frac{2\left(x+v^{2}\right)}{\gamma_{0}(1+v)}} d v\right) d z .
\end{aligned}
$$

2) Linearly Precoded SU-MIMO Scheme: For a linearly precoded MIMO system (also referred to as closed loop MIMO scheme) using the linear MMSE receiver, with 2 antennas at both the transmitter and the receiver, the probability of the unified effective SINR of each user can be expressed as [14]

$$
F_{\Gamma_{u}}(\gamma)=\int_{0}^{\gamma} d v \frac{1}{\left(\rho_{1} \rho_{2}\right)^{3}} \exp \left(-\frac{v}{\rho_{1}}\right) \varphi(\gamma, v)
$$

where

$$
\begin{aligned}
& \varphi(\gamma, v)=\rho_{2}{ }^{3} v^{2}\left[1-\exp \left(-\frac{\gamma-v}{\rho_{2}(v+1)}\right)\right]+2 \rho_{1}{ }^{2} \rho_{2}{ }^{3} \\
& -2 \rho_{1} \rho_{2}{ }^{3} v \cdot\left[1-\exp \left(-\frac{\gamma-v}{\rho_{2}(v+1)}\right)\left(1+\frac{\gamma-v}{\rho_{2}(v+1)}\right)\right] \\
& -\rho_{1}{ }^{2} \rho_{2}{ }^{3} \exp \left(-\frac{\gamma-v}{\rho_{2}(v+1)}\right) \cdot\left(\left(\frac{\gamma-v}{\rho_{2}(v+1)}\right)^{2}+\frac{2(\gamma-v)}{\rho_{2}(v+1)}+2\right) .
\end{aligned}
$$

and $\rho_{j}=p_{j} / N_{0}$, where $p_{j}$ is the power allocated to the $j$ th established sub-stream of the $i$ th $\mathrm{MS}$ and $N_{0}$ is the noise variance. By differentiating the distribution function expressed by (5), the PDF of the SINR for each user in the linearly precoded SDM SU-MIMO system can be derived as

$$
f_{\Gamma_{u}}(\gamma)=\int_{0}^{\gamma} \frac{\left(\rho_{2} v-\frac{\gamma-v}{1+v} \rho_{1}\right)^{2}}{\left(\rho_{1} \rho_{2}\right)^{3}(1+v)} \exp \left(-\frac{v}{\rho_{1}}-\frac{\gamma-v}{\rho_{2}(1+v)}\right) d v .
$$

Based on the SINR distribution shown in (5) and (6), we can derive its average achievable per-user rate from (2), as

$$
\begin{aligned}
\bar{R}= & \int_{0}^{\infty}\left[\int_{z}^{\infty} R(x)\left(\int_{0}^{x} \frac{\left(\rho_{2} v-\frac{x-v}{1+v} \rho_{1}\right)^{2}}{\left(\rho_{1} \rho_{2}\right)^{3}(1+v)} \exp \left(-\frac{v}{\rho_{1}}-\frac{x-v}{\rho_{2}(1+v)}\right) d v\right) d x\right] \\
& \cdot\left(K_{T}-1\right)\left[\int_{0}^{z} \frac{1}{\left(\rho_{1} \rho_{2}\right)^{3}} \exp \left(-\frac{v}{\rho_{1}}\right) \varphi(z, v) d v\right]^{K_{T}-2} \\
& \cdot\left(\int_{0}^{z} \frac{\left(\rho_{2} v-\frac{z-v}{1+v} \rho_{1}\right)^{2}}{\left(\rho_{1} \rho_{2}\right)^{3}(1+v)} \exp \left(-\frac{v}{\rho_{1}}-\frac{z-v}{\rho_{2}(1+v)}\right) d v\right) d z
\end{aligned}
$$

\section{B. Average Achievable Per-user Rate in MU-MIMO Scheme}

Unlike the SU-MIMO system, the multi-user MIMO system can serve $n_{t}$ users simultaneously, where $n_{t}$ is the number of transmitter antennas. The scheduler in base station selects at most $n_{t}$ users from the $K_{T}$ active users in the cell for data transmission in a time-slot, one user for each sub-stream. Thus, the number of competing users in such case is $\left(K_{T}-n_{t}\right)$.
1) Open Loop MU-MIMO Scheme: With a ZF receiver, the SINR on the $k$ th sub-stream $\Gamma_{k}$ has a Chi-squared PDF [13]

$$
f_{\Gamma_{k}}(\gamma)=\frac{n_{t} \sigma_{k}^{2} e^{-n_{t} \gamma \sigma_{k}^{2} / \gamma_{0}}}{\gamma_{0}\left(n_{r}-n_{t}\right) !}\left(\frac{n_{t} \gamma \sigma_{k}^{2}}{\gamma_{0}}\right)^{\left(n_{r}-n_{t}\right)},
$$

where $\gamma_{0}=E_{s} / N_{0}, E_{s}$ is the average transmit symbol energy per antenna and $N_{0}$ is the power spectral density of the additive white Gaussian noise, and $\sigma_{k}^{2}$ is the $k$ th diagonal entry of $\mathbf{R}_{\mathbf{t}}{ }^{-\mathbf{1}}$ where $\mathbf{R}_{\mathbf{t}}$ is the transmit-side spatial correlation covariance matrix. For the flat Rayleigh fading channels with uncorrelated receive antennas and uncorrelated transmit antennas, $\mathbf{R}_{\mathbf{t}}$ becomes an identity matrix, therefore, $\sigma_{k}^{2}=1$ in (8).

In the case of $n_{r}=n_{t}$, the CDF can be written in a closed form as

$$
F_{\Gamma_{k}}(\gamma)=1-e^{-\frac{n_{t} \gamma}{\gamma_{0}}}
$$

Because all of the users have identical and independent distribution of SINR, $Z$ represents the maximum SINR of all other competing users, the CDF of $Z$ can be expressed as

$$
F_{Z}(z)=\left[F_{\Gamma_{k}}(z)\right]^{K_{T}-n_{t}} .
$$

So we can derive the value of $\bar{R}$ as:

$$
\begin{aligned}
\bar{R} & =\int_{0}^{\infty}\left[\int_{z}^{\infty} \log _{2}(1+x) f_{\Gamma_{k}}(x) d x\right] \\
& \cdot\left(K_{T}-n_{t}\right)\left[F_{\Gamma_{k}}(z)\right]^{K_{T}-n_{t}-1} f_{\Gamma_{k}}(z) d z .
\end{aligned}
$$

Assume $n_{t}=n_{R}=2$, and substitute the closed form (9) into (11), the average achievable per-user rate in the open loop MU-MIMO scheme can be derived as

$$
\begin{aligned}
\bar{R} & =\int_{0}^{\infty}\left[\int_{z}^{\infty} \log _{2}(1+x) \frac{2}{\gamma_{0}} e^{-\frac{2 x}{\gamma_{0}}} d x\right] \\
& \cdot\left(K_{T}-2\right)\left[1-e^{-\frac{2 z}{\gamma_{0}}}\right]^{K_{T}-3} \cdot \frac{2}{\gamma_{0}} e^{-\frac{2 z}{\gamma_{0}}} d z .
\end{aligned}
$$

2) Linearly Precoded MU-MIMO Scheme: In a linearly precoded MU-MIMO systerm, the SINR distributions of the users in different sub-streams are different, the PDF of the SINR of each user in the $i$ th sub-stream can be expressed as [14]

$$
f_{\Gamma_{i}}(\gamma) \simeq \frac{1}{\rho_{i}} \frac{1}{[\beta(i)-1] !} \frac{\left(\gamma / \rho_{i}\right)^{\beta(i)-1}}{\tilde{\lambda}_{i}^{\beta(i)}} \exp \left(-\gamma /\left(\rho_{i} \tilde{\lambda}_{i}\right)\right),
$$

where $\rho_{i}=p_{i} / N_{0}, p_{i}$ is the power allocated to the $i$ th established sub-stream, and $N_{0}$ is the noise variance. $\lambda_{i}$ is the $i$ th largest non-zero eigenvalue of the matrix $\mathbf{H}_{i} \mathbf{H}_{i}^{H}$. Meanwhile, $\beta(i)=\left(n_{t}-i+1\right)\left(n_{r}-i+1\right)$, and $\tilde{\lambda}_{i}=\bar{\lambda}_{i} / \beta(i)=$ $\left[\int_{0}^{\infty} \lambda_{i} f_{\Lambda}\left(\lambda_{i}\right) d \lambda_{i}\right] / \beta(i)$.

Consequently, we can derive the CDF of the SINR of each user in the $i$ th sub-stream as

$$
\begin{aligned}
\operatorname{Pr}\left(\Gamma_{i} \leq \gamma\right) & =\int_{-\infty}^{\gamma} f_{\Gamma_{i}}(\alpha) d \alpha \\
& \simeq 1-\sum_{j=0}^{\beta(i)-1} \frac{\left(\gamma /\left(\rho_{i} \tilde{\lambda}_{i}\right)\right)^{j}}{j !} \exp \left(-\gamma /\left(\rho_{i} \tilde{\lambda}_{i}\right)\right) .
\end{aligned}
$$


For simplicity, we consider a $2 \times 2$ antenna configuration, and assume that the two sub-streams have identical distribution. Furthermore, we assume all the users have an identical and independent SINR distribution, thus the PDF of $Z$ which represents the maximum SINR of the competing $\left(K_{T}-2\right)$ users is given by

$$
f_{Z}(z)=\left(K_{T}-2\right)\left[F_{\Gamma_{1}}(z)\right]^{K_{T}-3} f_{\Gamma_{1}}(z) .
$$

Consequently, we can obtain $\bar{R}$ in the linearly precoded MU-MIMO system from (1) as

$$
\begin{aligned}
& \bar{R}=\int_{0}^{\infty}\left[\int_{z}^{\infty} \log _{2}(1+x) f_{\Gamma_{1}}(x) d x\right] \\
& \cdot\left(K_{T}-2\right)\left[F_{\Gamma_{1}}(z)\right]^{K_{T}-3} f_{\Gamma_{1}}(z) d z .
\end{aligned}
$$

Substituting (13) and (14) into (15), the average achievable per-user rate can be derived as

$$
\begin{aligned}
\bar{R} & =\int_{0}^{\infty}\left[\int_{z}^{\infty} \log _{2}(1+x) \frac{\left(x / \rho_{1}\right)^{\beta(1)-1}}{\rho_{1}[\beta(1)-1] ! \tilde{\lambda}_{1}^{\beta(1)}} \exp \left(-x /\left(\rho_{1} \tilde{\lambda}_{1}\right)\right)\right. \\
& \cdot\left(K_{T}-2\right)\left[1-\sum_{j=0}^{\beta(1)-1} \frac{\left(z /\left(\rho_{1} \tilde{\lambda}_{1}\right)\right)^{j}}{j !} \exp \left(-z /\left(\rho_{1} \tilde{\lambda}_{1}\right)\right)\right]^{K_{T}-3} \\
& \cdot \frac{1}{\rho_{1}[\beta(1)-1] !} \frac{\left(z / \rho_{1}\right)^{\beta(1)-1}}{\tilde{\lambda}_{1}^{\beta(1)}} \cdot \exp ^{\left(-z /\left(\rho_{1} \tilde{\lambda}_{1}\right)\right)} d z .
\end{aligned}
$$

\section{Unsaturated Case}

In the previous section, we analyzed the average achievable per-user rate $\bar{R}$, and found that $\bar{R}$ is a function of $K_{T}$, the number of busy users in the system. Now we consider unsaturated case where users have no data to transmit at random periods, i.e., user buffers could be empty for some periods of time.

We define $I$ as the number of users in busy state, and variable $p$ as the probability that a user is in the idle state. Then the probability mass function of $I$ can be derived as

$$
\operatorname{Pr}\{I=i\}=\left(\begin{array}{c}
K_{T} \\
i
\end{array}\right)(1-p)^{i} p^{K_{T}-i} .
$$

Hence, at the time of scheduling, there are actually $I$ users competing for the channel. In such a case, the average achievable per-user rate $\bar{R}$ could be written as $\bar{R}(i)$. The average achievable rate for each busy user, i.e., the rate at which the user can transmit data, is given by

$$
\begin{aligned}
\widetilde{R} & =\sum_{i=1}^{i=K_{T}} \bar{R}(i) \operatorname{Pr}\{I=i\} \\
& =\sum_{i=1}^{i=K_{T}} \bar{R}(i)\left(\begin{array}{c}
K_{T} \\
i
\end{array}\right)(1-p)^{i} p^{K_{T}-i} .
\end{aligned}
$$

Based on (17), system can estimate the transmission rate that can offer to each busy user. Meanwhile, by comparing this rate with user's requirement, an admission control principle can be set up: the user will be admitted when the average per-user rate satisfies its requirement.

\section{Analytical And Numerical Results}

We consider the case with 2 antennas at the transmitter and 2 receive antennas at the MS for the SU-MIMO case, and single antenna at the MS for the MU-MIMO case. First, we give the results of the average achievable per-user rate in saturated senario, including the uncoded and linearly precoded cases, in both SU-MIMO and MU-MIMO systems for LTE downlink transmission. Then we evaluate the unsaturated case in each MIMO system, assuming two different idle probabilities, i.e., $p$ equal to 0.3 and 0.6 .

Fig. 1 shows the average achievable per-user rate in the saturated case. Eq. (4) and (7) are used to calculate the peruser rate for the SU-MIMO scheme, in the uncoded case and precoded case respectively; (12) and (16) are used to calculate the per-user rates of uncoded and precoded cases for the MUMIMO scheme. This figure shows that the average per-user rate decreases when the number of total users increases. As we $x$ know, the total throughput increases when the UDO increases due to multiuser diversity, which is the traditional view when analyzing the system performance. But the resource that the system can offer to each user becomes less, especially when the number of users is large. Meanwhile, it shows that the average per-user rate in the SU-MIMO scheme is larger than the MU-MIMO scheme, given the same number of users in both cases. The reason is that two MSs are grouped together to form a virtual MIMO between the MSs and the BS in the MU-MIMO scheme. Each user only receives the signal from one antenna of BS. However, in the SU-MIMO case the user will obtain multiplexing gain.

Figs. 2 and 3 compare the average achievable per-user rate in the uncoded and linearly precoding cases, as well as the saturated and unsaturated cases, for SU-MIMO and MUMIMO, respectively. It can be seen that linearly prcoding provides higher rates for each user, i.e. better system performance, than the uncoded case. By comparing the saturated with the unsaturated senario, we can see that the achievable rate for each user in the unsaturated case is much higher, which means the busy users have more resource to transmit their data. In other words, when taking the idle state of users into consideration, the system could offer higher rate for each busy user, i.e., support higher QoS requirements of users in practice. When introduing the rate-based admission control as in [5], more users can be admitted into the system because of the higher rate provided. Finally, it shows that the increase in the idle possibility $p$ will greatly increase the achievable perrate in every case. Usually, the value of $p$ can be estimated by the statistical data of the system. The system can make a conservative strategy by decreasing the idle possibility $p$ slightly, or progressive strategy by increasing the value of $p$.

\section{Conclusions}

In this paper, we investigated the downlink transmission for both SDM SU-MIMO and MU-MIMO schemes. We derived mathematical expressions of SINR distributions for both uncoded and linearly precoded cases, and the average achievable per-user rate based on the corresponding SINR distribution. 


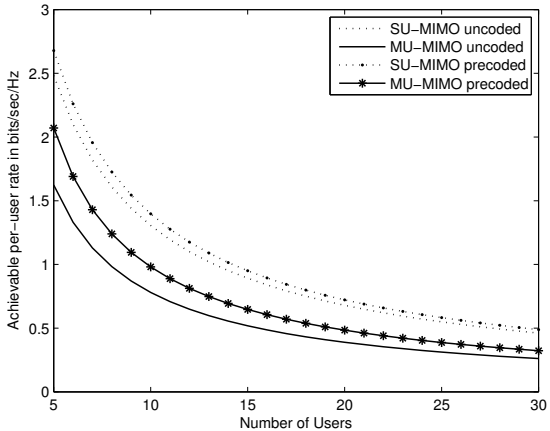

Fig. 1. Comparation of the achievable average per-user rate in SU-MIMO with MU-MIMO scheme.

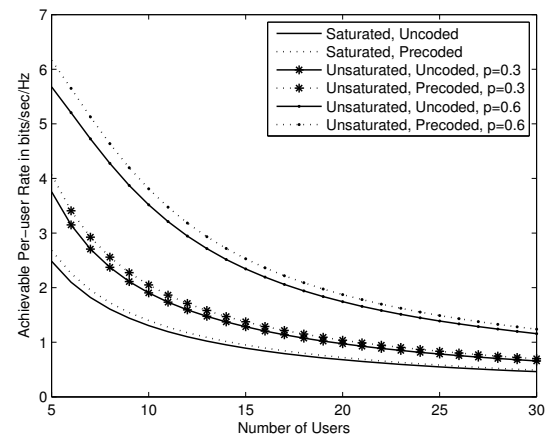

Fig. 2. Achievable average per-user rate for SDM SU-MIMO scheme vs.the number of users.

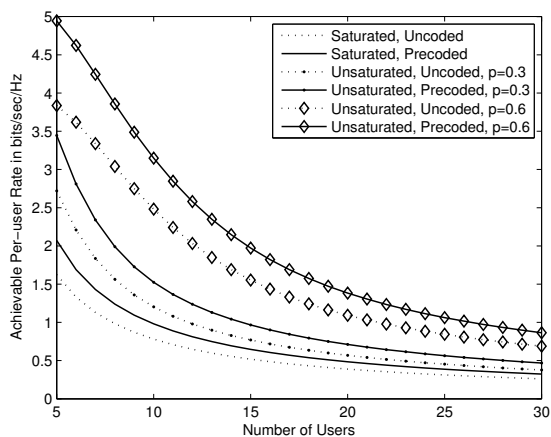

Fig. 3. Achievable average per-user rate for SDM MU-MIMO scheme vs.the number of users.

From the numerical results, it has been shown that the wireless resource which a system offers to each user decreases when the number of users increases. This result indicates that only a limit number of users can be admitted into the system in order to keep each user's QoS, although the multiuser diversity may improve the system capacity. Our investigation also reveals that the linearly precoding system achieves better performance in terms of achievable per-user rate compared to the open loop system without precoding. Meanwhile, a ratebased admission control principle was proposed by comparing the user's requirement with the transmission rate that the system offers.

\section{REFERENCES}

[1] 3GPP TR 25.814 V7.0.0, "Physical Layer Aspects for Evolved UTRA," Tech. Rep., June 2006.

[2] N. Wei, A. Pokhariyal, T. B. Sorensen, T. E. Kolding, and P. E. Mogensen, "Performance of MIMO with Frequency Domain Packet Scheduling in UTRAN LTE downlink," in IEEE VTC, Apr. 2007, pp. 1177-1181.

[3] Z. Lin, P. Xiao, Y. Wu, "Performance Analysis for Linearly Precoded Downlink Multiuser MIMO," EURASIP Journal on Wireless Communications and Networking, Article ID 797840, 12 pages, doi:10.1155/2011/797840.

[4] J. Zhang, Y. Wu, M. Xu, and J. Wang, "Linear transmitter precoding design for downlink of multiuser MIMO system," Electronics Letters, vol. 41 , no. 14 , July 2005

[5] M. Dianati, R. Tafazolli, X. Shen, and K. Naik, "Call admission control with opportunistic scheduling scheme," Wireless Communications and Mobile Computing, vol. 10, no. 3, pp.372-382, Mar. 2010.

[6] T. Park, O.-S. Shin, and K. B. Lee, "Proportional Fair Scheduling for Wireless Communication with Multiple Transmit and Receive Antennas," in IEEE VTC, Florida, USA, October 2003, vol. 3, pp. 1573-1577.

[7] J. M. Holtzman, "CDMA forward link waterfilling power control," in IEEE Vehicular Technology Conference (VTC), May 2000, pp. 16361667, Toyko, Japan.

[8] N. Wei, T. B. Sorensen, T. E. Kolding, and P. E. Mogensen, "Analysis and Evalution of Link Adaptation with MIMO adaptation," in IEEE VTC, Sept. 2006, pp. 1-5.

[9] T. K. Y. Lo, "Maximum ratio transmission," IEEE Trans. Commun., vol. 47, no. 10, pp. 1458-1461, Oct. 1999.

[10] 3GPP TR 25.214 V4.4.0, "Physical Layer Procedures (FDD)," Tech. Rep., Mar. 2002.

[11] P. Xiao, Z. Lin, A. Fagan, B. Vucetic, C. Cowan and Y. Wu "FrequencyDomain Equalization Technique for OFDMA Based Multiuser MIMO Systems with Improper Modulations," EURASIP Journal on Advances in Signal Processing, Article ID 645024, 2011.

[12] D. P. Palomar, J. M. Cioffi, and M. A. Lagunas, "Joint Tx-Rx Beamforming Design for Multicarrier MIMO Channels: A Unified Framework for Convex Optimization," IEEE Transaction on Signal Processing, vol. 51, no. 9, pp. 2381-2401, Sept. 2003.

[13] D. A. Gore, Jr. R. W. Heath, and A. J. Paulraj, "Transmit Selection in Spatial Multiplexing Systems," IEEE Communications Letters, vol. 6, no. 11, pp. 491-493, Nov. 2002

[14] Z. Lin, T. B. Sorensen and P. E. Mogensen, "Downlink SINR distribution of linearly precoded multi-user MIMO systems," IEEE Communications Letters, vol. 11, no. 11, pp. 850-852, Nov. 2007. 